\title{
Conditioned Response Timing and Integration in the Cerebellum
}

\author{
John W. Moore ${ }^{1}$ and June-Seek Choi \\ Neuroscience and Behavior Program \\ University of Massachusetts \\ Amherst, Massachusetts 01003
}

\begin{abstract}
Classical conditioning procedures instill knowledge about the temporal relationships between events. The unconditioned stimulus (US) is the event to be timed. The conditioned response (CR) is viewed as a prediction of the imminence of the US. Knowledge of the elapsed time between conditioned stimuli (CSs) and US delivery is expressed in the topological features of the CR. The peak amplitude of the CR coincides with the timing of the US. A simple connectionist network based on Sutton and Barto's Time Derivative (TD) Model of Pavlovian Reinforcement provides a mechanism that can account for and simulate CR timing in a variety of protocols. This article describes extensions of the model to predictive timing under temporal uncertainty. The model is expressed in terms of equations that operate in real time according to a competitive learning rule. The unfolding of time from the onsets and offsets of events such as CSs is represented by the propagation of activity along a sequence of time-tagged elements. The model can be aligned with anatomical circuits of the cerebellum and brain stem that are essential for learning and performance of conditioned eye-blink responses.
\end{abstract}

\section{Introduction}

Accumulating evidence indicates that the cerebellum mediates learning and performance of

${ }^{1}$ Corresponding author. classically conditioned responses (CRs). Lesions or inactivation of components of neural circuits involved in performance of conditioned eye-blink responses (Fig. 1) ${ }^{1}$ have been shown to disrupt the timing and amplitude of eye-blink CRs (Raymond et al. 1996; Thompson and Krupa 1994). There is growing evidence that these operations prevent learning when applied to either the cerebellar cortex (Gruart and Yeo 1995) or deep nuclei (Ramnani and Yeo 1996).

The lesion and inactivation evidence has been supported by recording studies that have demonstrated neuronal activity correlated with the timing and amplitude of eye-blink CRs. For example, a series of studies by Moore and his associates have shown neuronal firing patterns to be predictive of CRs in each structure of the efferent pathway, from the putative site of learning in the cerebellar cortex to motoneurons. The relevant portion of the cerebellar cortex for eye-blink conditioning in rabbit is Larsell's hemispheral lobule VI (HVI), which corresponds to the simplex lobe in cat. Berthier and Moore (1986) recorded from Purkinje cells (PCs) in HVI; Berthier and Moore (1990) recorded from deep cerebellar nucleus interpositus (IP); Desmond and Moore (1991a) recorded from the red nucleus (RN); Richards et al. (1991) recorded from the spinal trigeminal nucleus pars oralis (SpO). The results of these studies are consistent with recording studies carried out in other laboratories (for review, see Thompson and Krupa 1994). In sum, the activity of neurons within these structures mir-

${ }^{1}$ Figure 1 does not depict projections for the pontine nuclei (PN) to deep cerebellar nucleus interpositus (IP) that some investigators have proposed (e.g., Raymond et al. 1996). These projections have been omitted because their existence has not been confirmed in our laboratory and because they are not incorporated into the cerebellar implementation presented later on. Figure 1 also does not depict projections to $I P$ from the inferior olive $(\mathrm{IO})$ and the lateral reticular nucleus, which we have confirmed, because they are not incorportated into the implementation scheme.

LEARNING \& MEMORY 3:116-129 @ 1997 by Cold Spring Harbor Laboratory Press ISSN1072-0502/97 \$5.00

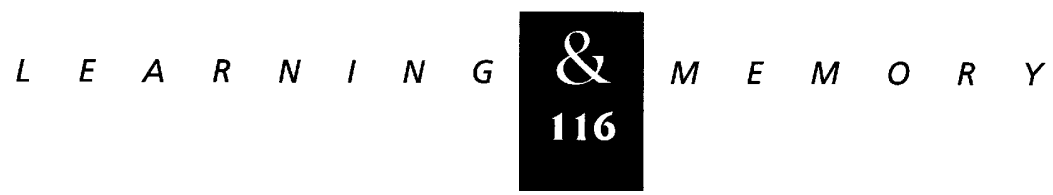




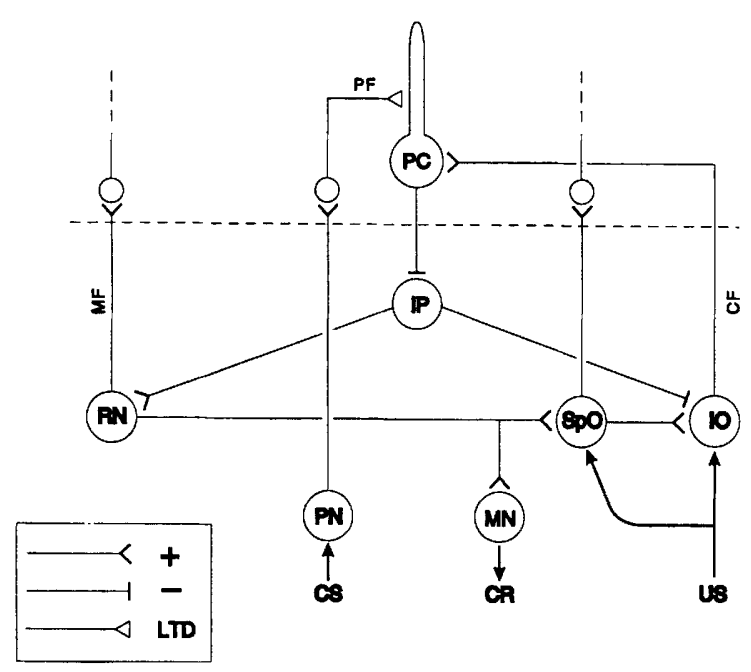

Figure 1: Cerebellar and brain stem circuits underlying eye-blink conditioning (after Rosenfield and Moore 1995). (MF) mossy fibers; (PF) parallel fibers; (PC) Purkinje cell; (RN) red nucleus; (IP) interpositus nucleus; (SpO) spinal trigeminal nucleus pars oralis; (CF) climbing fiber; (IO) inferior olivary nucleus; $(\mathrm{PN})$ pontine nucleus; (MN) motoneurons; (LTD) long-term depression; (CS) conditioned stimulus; (CR) conditioned response; (US) unconditioned stimulus.

ror CRs as they unfold in time, encoding their latency and peak amplitude. How does this come about?

There are several ways to approach this question. The one we have favored relies on the development of computational models that can be expressed as neural networks. This involves three steps: (1) Devise real-time computational models that describe as much of the known behavioral and physiological evidence as possible; (2) devise an implementation scheme that aligns features of the model with involved neural circuits; and (3) test implications of the model and its implementation in novel experiments.

This article applies these steps to the Time Derivative (TD) Model of Pavlovian Reinforcement (Sutton and Barto 1990). Before presenting the TD model and a plausible implementation within the cerebellum, we review related computational models and their limitations. Next, we present the TD model and illustrate its predictions in simple and complex paradigms. Simple paradigms are those that involve training with a single conditioned stimulus (CS) presented repeatedly with an unconditioned stimulus (US) at a fixed CS-US interval (interstimulus interval, or ISI). Complex paradigms are those involving integration of information from two or more types of training trials. Familiar examples of complex paradigms include Kamin blocking, conditioned inhibition, mixed CS-US interval training (temporal uncertainty), and higherorder conditioning.

\section{Theoretical Background}

Contemporary computational models of learning in classical conditioning trace their origins to the Rescorla-Wagner (RW) model (Rescorla and Wagner 1972). The RW model supplanted earlier mathematical models, such as the statistical learning theory, because of its ability to generate accurate predictions in a number of complex conditioning paradigms, including Kamin blocking and conditioned inhibition. The RW model can be expressed by an equation that specifies how the associative connection $\left(V_{i}\right)$ between the $i$ th CS and the $\mathrm{CR}$ is modified from one trial $(t-1)$ to the next $(t)$ as a function of pairing with a US.

$$
\Delta V_{i}(t)=\beta[\lambda(t)-Y(t-1)] \times \alpha X_{i}(t)
$$

where

$$
Y(t)=\sum_{j} V_{j}(t) X_{j}(t)
$$

The subscript $j$ indexes all CSs that are active on trial $t, \alpha$ and $\beta$ are rate parameters $(0<\alpha, \beta \leqslant 1), \lambda(t)$ is the strength of the US, and $X_{i}(t)$ denotes the presence $\left[X_{i}(t)=1\right]$ or the absence $\left[X_{i}(t)=0\right)$ of $\mathrm{CS}_{\mathrm{i}}$.

The RW model features a "competitive" learning rule. This means that connection weights are adjusted so that the sum of the values of all CSs present on a trial approach the strength of the US, $\lambda$. Kamin blocking occurs whenever a new CS is combined with one that had been previously trained. Blocking occurs because the previously trained CS fully predicts the US, having garnered most of the associative value the US can support. Conditioned inhibition occurs whenever a CS is combined with a previously trained excitatory CS but not paired with the US. Both CSs lose associative value, but because the added CS is never paired with the US, its value becomes increasingly negative. The net effect of combining an excitatory and inhibitory CS after training in this paradigm is a marked suppression or cancellation of the CR.

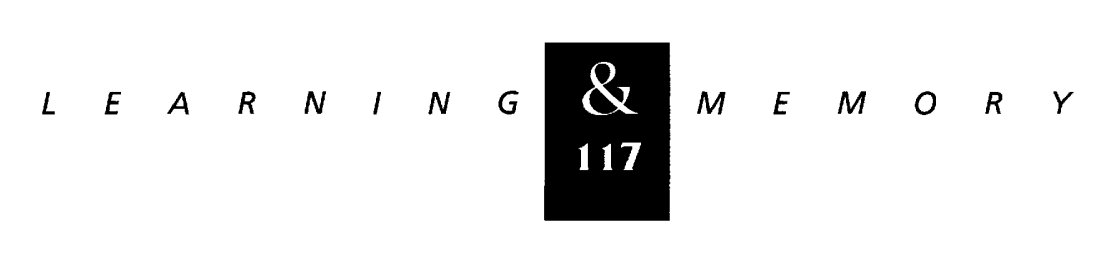


Although the RW model and other trial-level models continue to impact learning theory in many domains, their limitations motivated the emergence of real-time models of classical conditioning (Klopf 1988). Two limitations of trial-level models are their inability to generate CS-US interval functions or higher-order conditioning (Sutton and Barto 1990). Real-time models invoke other processes to account for these phenomena. In realtime models, the timing and duration of CSs and the US determine how learning unfolds. Real-time models imply that time is treated as a continuous variable and that learning can therefore be expressed by differential equations in time. In practice (and for simulation purposes), real-time models segment time into a series of computational epochs with a fine enough grain to capture dynamical events on a time scale appropriate for the preparation.

Sutton and Barto's (SB) Time Derivative (TD) model was the first real-time model to generate CS-US interval functions and higher-order conditioning (Sutton and Barto 1981; Moore et al. 1986). The SB model is a member of a class of models that Sutton and Barto (1990) have referred to as $\dot{Y}$ theories of learning. These theories adjust connection weights (associative values) according to the first time derivative of the learning system's output or response, $Y(t)$. If the output on the current time step is greater than that of the preceding time step, active connections are strengthened. If the output on the current time step is less than that of the preceding time step, active connections are weakened. The US affects learning only to the extent that it contributes to output.

$$
\Delta V_{i}=\beta \dot{Y} \times \alpha_{i} \bar{X}_{i}
$$

where

$$
\dot{Y}=Y(t)-Y(t-1)
$$

Any device that would implement $\dot{Y}$ learning must receive information about $Y(t)$ and $Y(t-1)$.

The following three equations specify the SB model.

$$
\Delta V_{i}(t)=\beta[Y(t)-Y(t-1)] \times \alpha \bar{X}_{i}(t)
$$

where

$$
Y(t)=\sum_{j} V_{j}(t) X_{j}(t)+\lambda(t)
$$

$\bar{X}_{i}(t)$ specifies the eligibility of the $i$ th connection for modificaiton. A CS's eligibility decreases geometrically after offset according to the following equation:

$$
\bar{X}_{i}(t+1)=\bar{X}_{i}(t)+\delta\left[X_{i}(t)-\bar{X}_{i}(t)\right]
$$

A slowly decaying eligibility allows modification of connection weights for CSs that are no longer contributing to output.

Although the SB model provides a framework for describing aspects of eye-blink conditioning that the RW model cannot address, it does not generate realistic-appearing CRs. There are two problems with the SB model in this regard. First, the model does not capture the fact that the onset of CRs is delayed with respect to CS onset and peaks at the time of the US. Second, the model predicts that response amplitude, $Y(t)$, in the presence of the US is too large (equation 6). For example, if CS elicits a $10-\mathrm{mm}$ eyelid closure after training, and if the US presented alone elicits a $10-\mathrm{mm}$ eyelid closure, then the combination of the CS and US should elicit a $20-\mathrm{mm}$ response. This does not happen because eyelids can close only so far and no further. Moore et al. (1986) devised a variant of the SB model that attempted to solve these problems. First, it was assumed that the onset of a CS does not trigger an immediate response but instead causes the gradual rise in amplitude that peaks at a fixed time and that is sustained until the CS is withdrawn. Second, equation 6 was modified so that the contribution of the US, $\lambda$, to $Y(t)$ decreases progressively as $V_{i}$ increases over training.

Despite these modifications, the SB model does not provide an adequate model for generating realistic CRs (Desmond 1990). For one thing, the SB model cannot describe the shifts in CR timing that occur when the CS-US interval is changed during training. Nor can it describe the fact that CRs can be elicited by stimulus offsets as well as stimulus onsets (Desmond and Moore 1991b). These problems with the SB model led to the development of another model, similar to SB, but with additional features that overcame the former's limitations (Desmond and Moore 1988; 1991b; Moore et al. 1989; Moore 1992; Moore and Desmond 1992). We refer to this model as VET, for associative values based on expectations about timing.

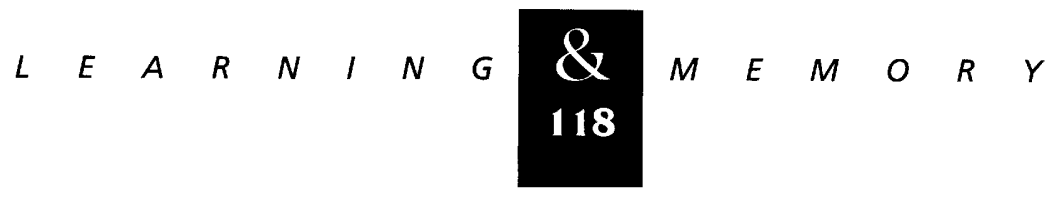


The VET model assumes that both stimulus onsets and offsets cause cascades of spreading activation. This spreading activation sequentially engages time-tagged "input elements". These input elements can be regarded as serial components of the nominal CS (Sutton and Barto 1990). ${ }^{2}$ Computations on these sequentially activated elements give rise to CRs that mirror the CS-US intervals employed in training. Such CRs are said to be temporally adaptive. The timing structure of the VET model can be applied to the TD model, as illustrated later on.

Although it is an advance on the SB model, the VET model has one important deficiency. Like the RW model (but unlike the SB model), it cannot generate higher-order conditioning. That is, it does not allow for the establishment of a CR when a novel stimulus is paired with a previously trained CS. The SB model can generate higher-order conditioning because the strength of conditioning (i.e., the value or weight of the connection between CSs and the CR) depends only on the changes in output from one time step to the next. Increases in output result in increases in connection weight; decreases in output result in decreases in connection weight. The US is not essential for this learning. In contrast, changes in connection weights in VET (and RW model) revolve about the US. Weights increase when the US is presented and decrease when it is omitted, subject to the constraints of competitive learning.

\section{Hybrid Learning: TD Model}

Experience with the SB and VET models suggested the need for a model that combines the best features of both. Sutton and Barto (1990) showed that the TD model overcomes the limitations of the SB and VET models. We have extended the TD model by incorporating the timing structure of the VET model. The TD learning rule is given by the following equation:

$$
\Delta V_{i}(t)=\beta[\lambda(t)+\gamma Y(t)-Y(t-1)] \times \alpha \bar{X}_{i}(t)
$$

\footnotetext{
${ }^{2}$ Sutton and Barto (1990) refer to this timing structure as a complete serial compound (CSC). The TD model with the CSC representation of time has been applied to predictive timing and error correction in the dopaminergic reward system of monkeys (Schultz et al. 1997).
}

where

$$
Y(t)=\sum_{j} V_{j}(t) X_{j}(t)
$$

As in the SB model, $\bar{X}_{i}$ refers to eligibility for modification.

$$
\bar{X}_{i}(t+1)=\bar{X}_{i}(t)+\delta\left[X_{i}(t)-\bar{X}_{i}(t)\right]
$$

The hybrid nature of the learning rule is evident in the fact that changes in connection weights depend on two reinforcement components. One component is contributed by the US, $\lambda$. The other component is contributed by a $\dot{Y}$ component, $\gamma$ $Y(t)-Y(t-1)$.

A key feature of the TD model is the parameter $\gamma(0<\gamma \leqslant 1) . \gamma$ is referred to as the discount parameter because $Y(t)$ is not known with certainty until after the fact. That is, $Y(t)$ must be estimated by using the connection weights computed on the previous time step, $Y(t)=\Sigma_{j} V_{j}(t-1) \times X_{j}(t) . \gamma$ can be regarded as the penalty for using $V(t-1)$ as an estimate of $V(t)$.

The TD model overcomes the deficiencies of earlier models while retaining their ability to describe complex paradigms such as Kamin blocking and conditioned inhibition. However, for the TD model to encompass CR timing and topography, Sutton and Barto (1990) proposed that the elapsed time between the onset of a CS and the US be segmented into an ordered sequence of serial components. These serial components are, for all intents and purposes, the same as the time-tagged input elements of the VET model. The subscript $i$ in the equations of the TD model refers to a single serial component.

Figure 2 shows simulated CRs with the TD model with variations of two parameters, $\gamma$, and $\delta$ ( $\lambda$ held constant). Both parameters contribute to the latency and amplitude of CRs. These simulated CRs are realistic in that they resemble goal gradients: Amplitude rises progressively to peak at the time of US onset.

For the TD model to describe CR timing and topography in trace conditioning and in complex paradigms involving multiple CSs, we adopt the timing structure of the VET model. Both CS onsets and offsets are assumed to trigger cascades of spreading activation. This spreading activation is mapped onto the serial components of the TD model. Each nominal CS, such as a tone and a light,

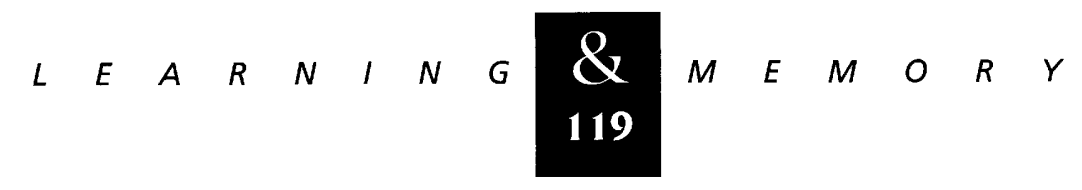



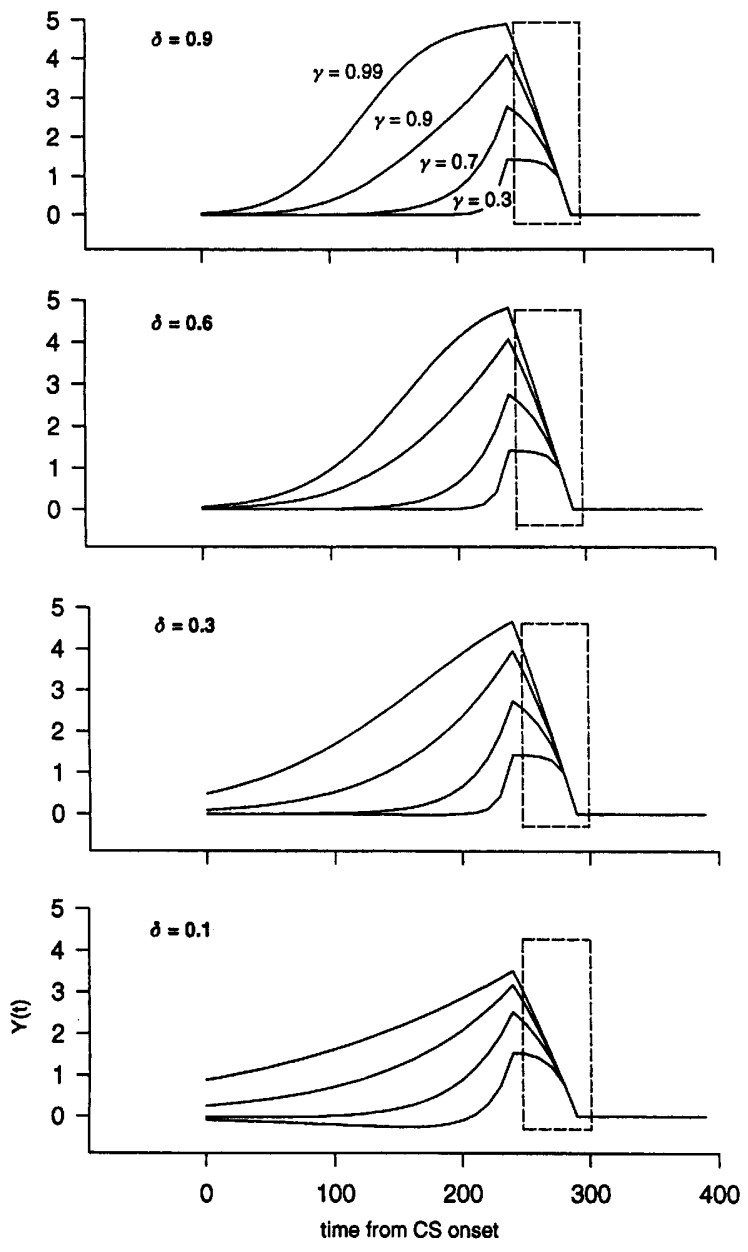

Figure 2: Simulated $C R s, Y(t)$, after 200 trials as a function of $\gamma$ and $\delta$. Time steps in this and other simulations are $10 \mathrm{msec}, \alpha=0.05, \beta=1.0$, and $\lambda=1.0$. The rectangle in each panel indicates the duration $(50 \mathrm{msec})$ and intensity of the US [scales in terms of $Y(t)]$. Note that CR timing is determined primarily by the discount factor, $\gamma$.

initiates an independent cascade that sequentially activates the variables $X_{i}$ in the model $(i=1,2,3, \ldots)$. The duration of activation of a serial component need not be fixed or constant, but for simulation purposes we have assumed a temporal grain of $10 \mathrm{msec}$. Hence, this is the assumed duration of activation of a serial component. When activated, $X_{i}=1$; when inactivated (after $10 \mathrm{msec}$ ), $X_{i}$ resets to a baseline of 0 as the next serial component, $X_{i+1}$ is activated. Although $X_{i}$ is no longer active and therefore no longer contributes to $Y$, the output or response, its connection to the output, $V_{i}$, remains eligible for modification over succeeding time steps. Eligibility decays at a rate determined by $\delta$. And, as mentioned, just as a nominal CS initiates a cascade of activation among serial components, so too does its offset. The two cascades are assumed to operate independently and in parallel. There are limits on how long these cascades might last, that is, on the number of sequentially activated elements in each cascade. The only requirement is that these cascades span the CS-US intervals employed in training.

\section{TD Model and Temporal Uncertainty}

Like the VET model, the TD model predicts the timing and amplitude of CR waveforms in complex training paradigms. One such paradigm involves training with a random mixture of two CS-US intervals. We refer to this training paradigm as conditioning under temporal uncertainty (Millenson et al. 1977). If the two CS-US intervals are sufficiently different (e.g., 300 vs. $700 \mathrm{msec}$ ), then rabbits learn to generate bimodal CRs with amplitude peaks at the temporal loci of the two times of US occurrence.

Figure 3 shows simulated bimodal CRs following temporal uncertainty training with CS-US intervals of 300 and $700 \mathrm{msec}$. In Figure 3A, the CS duration is $300 \mathrm{msec}$. The simulated bimodal CR to a CS-alone probe trial has two amplitude peaks. The one at $700 \mathrm{msec}$ is larger than the one at $\mathbf{3 0 0}$ msec because CS offset contributes to the 700msec peak but not the $300-\mathrm{msec}$ peak. In Figure $3 \mathrm{~B}$, the CS duration is $800 \mathrm{msec}$. Because CS offset occurs after the longest CS-US interval, its cascade does not contribute to the second peak. Consequently, the two peaks have the same amplitude.

\section{Uncertainty Training and the Cerebellum}

How is experience with temporal uncertainty represented in the cerebellum? Do firing patterns of single neurons express the timing and amplitude eye-blink CRs in a manner predicted by the TD model? We have begun to address this question in experiments with rabbits, employing the temporal uncertainty protocol shown in Figure 4A O.-S. Choi and J.W. Moore unpubl.). The CS is a $300-\mathrm{msec}$ tone. The US is a mild electric current applied to the periocular tissue of the right eye. Training consists of a random mixture of two trial types. On trial-type 1, the CS-US interval is $300 \mathrm{msec}$. On trial-type 2, the CS-US interval is $700 \mathrm{msec}$. After 20 daily sessions ( 80 trials/session with an average

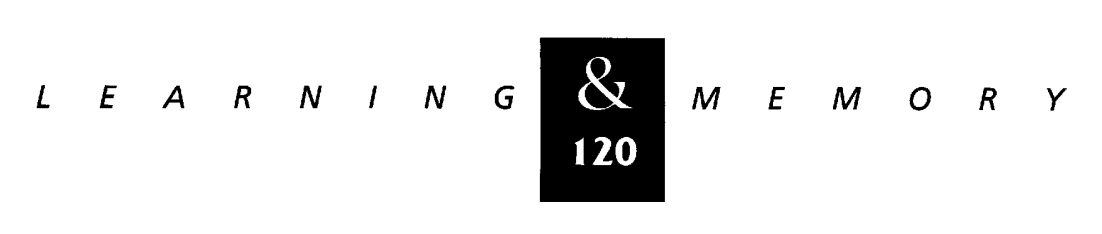


A
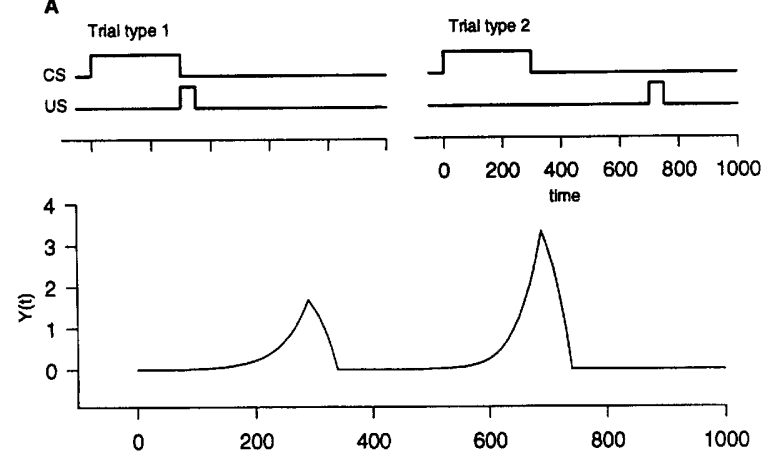

B
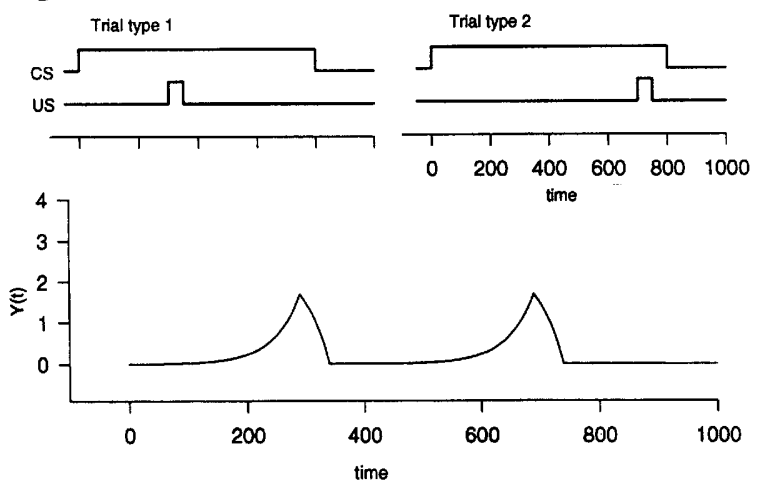

Figure 3: Simulated bimodal CRs following training with a random mix of two CS-US intervals. (A) Two types of training trials and a simulated bimodal $C R$ waveform. The CS is $300 \mathrm{msec}$ in duration. The CS-US interval is $300 \mathrm{msec}$ on trial type 1 and $700 \mathrm{msec}$ on trial type 2. Notice that the second CR peak is larger than the first CR peak. (B) Two types of training trials and a simulated CR waveform. The CS is $800 \mathrm{msec}$ in duration. The CS-US intervals are the same as in A. Note that the two CR peaks have the same amplitude.

intertrial interval of $25 \mathrm{sec}$ ), rabbits are surgically prepared for microelectrode recording (Berthier and Moore 1990). Training resumes follow recovery to ensure that bimodal CRs are well-established. A microelectrode is then advanced through the cerebellar cortex and into deep nucleus IP. During recording, the two trial types employed in training continue to be presented, but there are also CS-alone probe trials.

Figure $4 \mathrm{~B}$ shows a single $\mathrm{CS}$-alone probe trial (CS onset occurs at time $=350 \mathrm{msec}$ ). The top trace is a record of eyelid position as a function of time. Notice that there are two amplitude peaks and that these are located at the loci of the US. The second peak is larger than the first, in agreement with the TD model as shown in Figure 3A. The second trace shows the firing of an IP neuron on this trial. Note that the rate and duration of firing are highly related to the two CR peaks. Figure $4 \mathrm{C}$ shows the averaged CR topography (top trace) and spike histogram for all probe trials with this neuron.

Figure 4, D and E, shows averaged CR topographies and spike histograms for this neuron on reinforced trials (upward arrows mark the US). Figure $4 \mathrm{D}$ is interesting because it shows that the occurrence of the US cancels the second amplitude peak and terminates the CS-triggered spiking. This is an important observation because it suggests (but does not prove) that the US acts as a conditioned inhibitor. ${ }^{3}$ In terms of the model, the US initiates a cascade of activation of serial components that is never paired with the US. In training trials on which the US occurs at $300 \mathrm{msec}$ (trial type 1), the US-triggered cascade exists alongside two CS-triggered cascades, an onset cascade and an offset cascade. The CS-triggered cascades are paired with the US on trial type 2 (700-msec CS-US interval), so they are excitatory. In contrast, because there is only one US per trial, the US-triggered cascade is never paired with the US. According to competitive learning rules, serial components of the cascade triggered by the US become conditioned inhibitors and therefore they have the capacity to suppress CRs.

If this scenario is correct, it indicates that conditioned inhibition is expressed at the level of single cerebellar neurons. The expression of both excitation and inhibition within the same cerebellar neuron would be an important discovery about the locus of action of conditioned inhibition (Blazis and Moore 1991). Such an observation would be consistent with evidence that the cerebellum is the locus of extinction, the gradual decline of the CR through repeated presentations of a CS without reinforcement: (1) Ramnani and Yeo (1996) showed that reversible inactivation of nucleus IP protects against extinction; (2) Perrett and Mauk (1995) showed that vermal cerebellar lesions interfere with extinction, just as lesions of HVI interfere with $C R$ acquisition and performance.

Several investigators have suggested that cerebellar PCs mediate the CR-related activity ob-

${ }^{3}$ It is unlikely that the US simply terminates all timing cascades, thereby accounting for the absence of the second amplitude peak on 300-msec probe trials. Instead, this capacity develops progressively with training; the US does not terminate the second peak until training is well advanced.

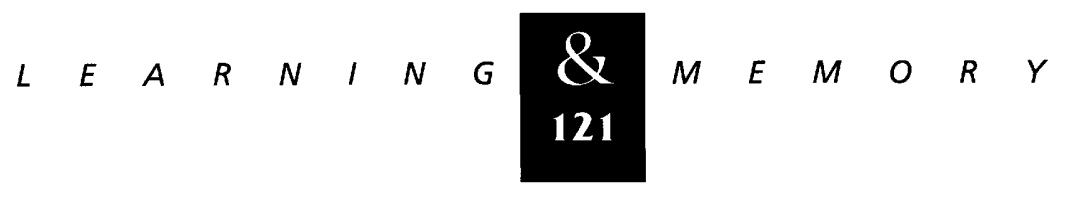


A
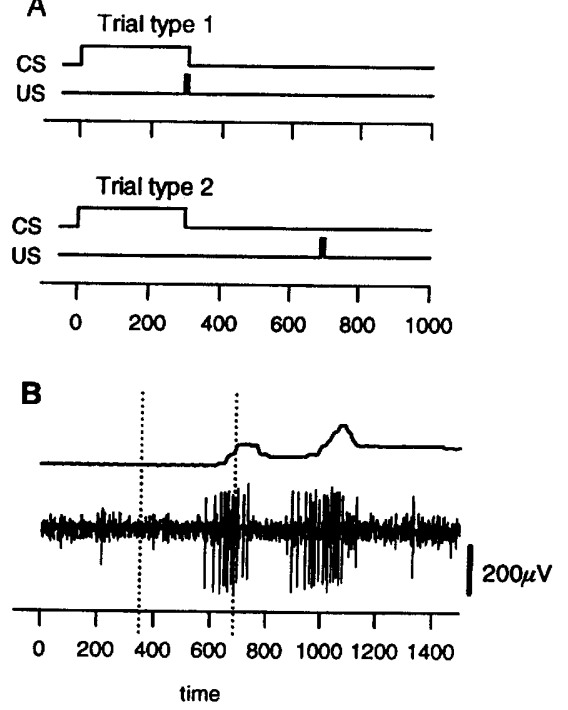

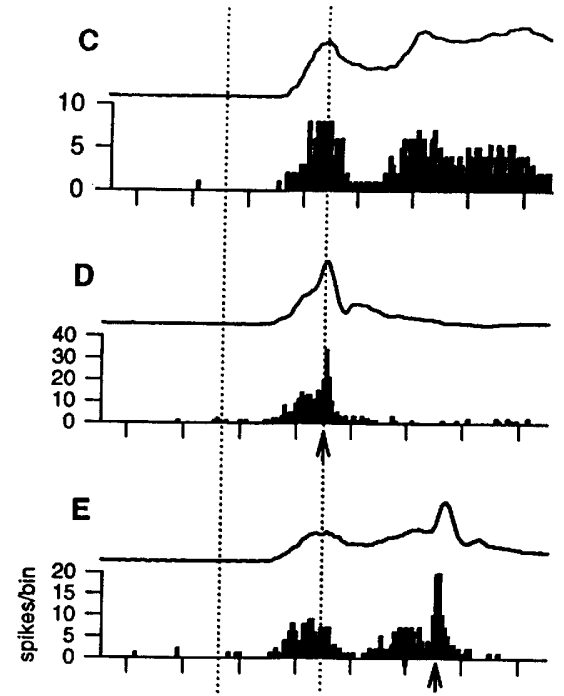

Figure 4: Firing patterns of an IP neuron related to bimodal CRs. (A) Two types of training trials: CS duration $=300 \mathrm{msec}$. CS-US intervals are $300 \mathrm{msec}$ (trial type 1) and $700 \mathrm{msec}$ (trial type 2). (B) A single CS-alone trial: Top trace shows CR waveform; second trace shows neuronal response. Vertical dotted lines mark CS onset and offset. $(C)$ Average CR waveforms and spike histogram on probe trials $(n=6)$. (D) Average response waveform and spike histogram on type 1 reinforced trials $(n=11)$. Arrows mark the US. (E) Average response waveform and spike histogram on type 2 reinforced trials $(n=8)$.

served in nucleus IP (Berthier and Moore 1986; Thompson 1986). ${ }^{4}$ Because PCs are inhibitory (Fig. 1), they can generate the CR-related increases in firing in IP neurons only by decreasing their rate of firing. Figure 5, which summarizes results from a PC in a different animal than the one in Figure 4, indicates that this may be the case. Figure $5 \mathrm{~A}$ shows a single probe trial following temporal uncertainty training in the protocol of Figure 4. The top trace shows two CR peaks, with the second peak larger than the first. The second trace shows pauses in simple spike rates related to each CR peak. Figure 5, shows the averaged CR waveforms and spike histogram for this cell on probe trials. Figure 5, C and D, shows averaged CR waveforms and spike histograms for this cell on the two reinforced trial types. The histograms show that the rate of simple spiking decreased in anticipation of each of the two amplitude peaks. The US caused a brief burst of activity that was followed by a postUS pause on the order of $100 \mathrm{msec}$. There is some indication of conditioned inhibition by the US in

\footnotetext{
${ }^{4}$ The activity of a PC expressing long-term depression (LTD) would not to be as highly correlated with eye-blink CRs as would the activity of an IP neuron, because of the many-toone convergence of PCs onto IP neurons (Ito 1984).
}

Figure $5 \mathrm{C}$, because of the transitory increase in spiking $100 \mathrm{msec}$ after the US. ${ }^{5}$

\section{Cerebellar Implementation of the TD Model}

Our recording studies indicate that the full complexity of conditioned eye blinks in a temporal uncertainty paradigm can be represented in the firing of single IP neurons. Furthermore, it is possible that this complexity is also captured in the activity of individual PCs (Fig. 5), as suggested by theorists (e.g., Moore et al. 1989; Fiala et al. 1996). From this perspective, IP neurons execute motor programs by inverting signals generated by PCs. We turn next to a consideration of how the TD model's learning rule might be implemented in the cerebellar cortex.

TD learning can be implemented in the cerebellum by aligning known anatomical ingredients with elements of the learning rule. In TD learning,

${ }^{5}$ Conditioned inhibition would be implemented by parallel fiber (PF)/PC synapses expressing long-term potentiation (LTP). It is not presently known whether LTP and LTD synapses can coexist on the same PC, as suggested by Fig. 5 .

$$
\begin{array}{llllllll}
L & E & A & R & N & I & N & G \\
\begin{array}{l}
\boldsymbol{Q} \\
122
\end{array} & M & E & M & O & R & Y
\end{array}
$$



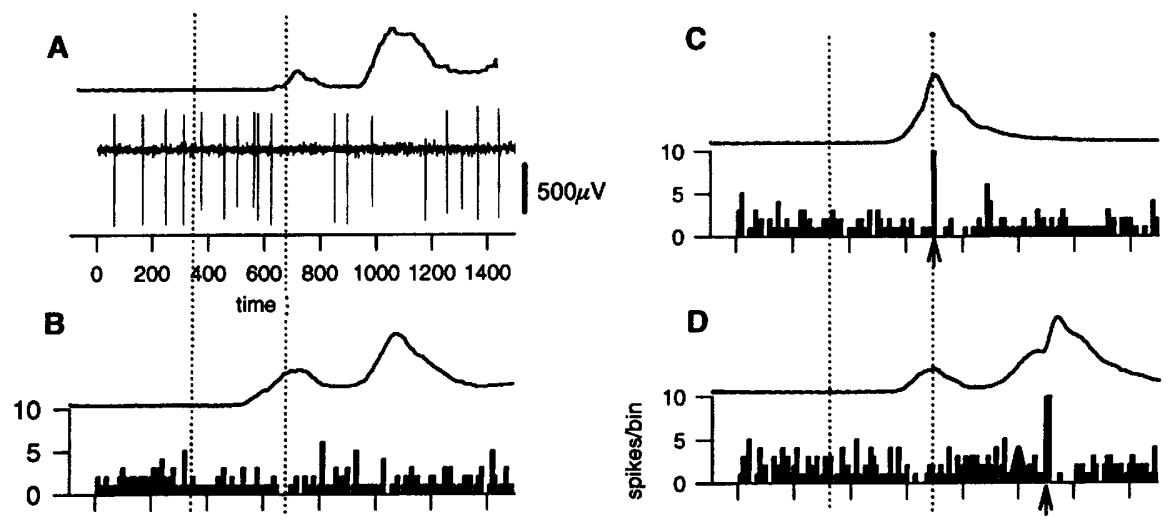

Figure 5: Firing patterns of a PC related to bimodal CRs. (A) A single CS-alone probe trial: Top trace shows CR waveform; second trace shows neuronal response. Vertical dotted lines mark CS onset and offset. (B) Average CR waveforms and spike histogram on probe trials $(n=13)$. (C) Average response waveform and spike histogram on type 1 reinforced trials $(n=11)$. (D) Average response waveform and spike histogram on type 2 reinforced trials $(n=14)$.

we assume that each computational time step after the onset or offset of a CS is represented by anatomically distinct inputs to the cerebellum. The onset or offset of a CS initiates a spreading pattern of activation among neurons tied to whatever sense modality is involved. This spreading of activation, possibly under entrainment from oscillators, engages pontine nuclear (PN) cells, the primary source of cerebellar mossy fibers (MFs), and their associated granule cells. Therefore, timing elements should be regarded as ensembles that include PN cells, MFs, granule cells, parallel fibers and influences from intrinsic cerebellar neurons such as Golgi cells. This may be why CR timing is disrupted by lesions of the cerebellar cortex (Perrett et al. 1993). Entrainment by oscillators might occur at the level of the PN, as these are the nexus of neural influences from the lemniscal systems, midbrain, and forebrain (Wells et al. 1989). Finegrain temporal segmentation might occur locally within the cerebellum, as proposed by Bullock et al. (1994). Coarse-grain temporal segmentation and coherence might occur globally with the participation of the hippocampus, as suggested by Grossberg and Merrill (1996).

The implementation relies on evidence from rabbit eye-blink conditioning that CR topography is formed in the cerebellar cortex through converging contiguous action of parallel fiber (PF) and climbing fiber (CF) input to PCs. This action produces synaptic long-term depression (LTD). Chen and Thompson (1995) and Schreurs et al. (1996) have demonstrated pairing-specific LTD of PCs in cerebellar slice preparations from rabbits, using pa- rameters that support conditioning in intact animals. Consistent with the LTD hypothesis, Hesslow (1994) showed that stimulation of the cerebellar cortex (HVI) inhibits eye-blink CRs in decerebrate cats. Mechanisms of LTD in the cerebellum have been spelled out in recent articles (Kano et al. 1992; Konnerth et al. 1992; Schreurs and Alkon 1993; Eilers et al. 1995; Ghosh and Greenberg 1995; Hartell 1996; Schreurs et al. 1996, 1997; Kim and Thompson 1997).

Figure 1 incorporates recent anatomical findings by Rosenfield and Moore (1995) indicating the existence of projections to HVI from the RN and SpO. CS information ascends to granule cells in the cerebellar cortex (Larsell's lobule HVI) via MFs originating in the PN. Information about the US ascends to the cerebellar cortex by two routes, MF projections from the sensory SpO in Figure 1, and CF projections from the inferior olive (IO). A CR is generated within deep cerebellar nucleus IP, where the CR is formed by modulation from PCs. A full-blown CR is expressed as an increased rate of firing among IP neurons (e.g., Berthier and Moore 1990; Berthier et al. 1991). This activity is projected to the contralateral RN. From RN, activity is projected to motoneurons (MNs) that innervate the peripheral musculature controlling the position and movements of the eyelids and eyeball (Desmond and Moore 1991a). The RN also projects to SpO, giving rise to CR-related activity among these neurons (Richards et al. 1991).

Figure 1 depicts an inhibitory projection from IP to IO. The consequence of this arrangement is that olivary signals to PCs are suppressed when the

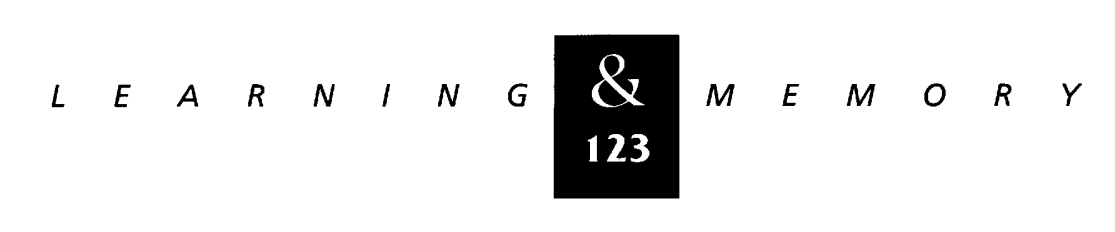


CR representation within IP is robust. This anatomical feature suggests that $\mathrm{CFs}$ are excited only when the US occurs and the CR is weak or absent. This scenario has been supported by Sears and Steinmetz (1991), who showed that neural activity recording within IO diminishes during CR acquisition. In the TD model, the $C R$ is regarded as a prediction of US onset. Therefore, a CR should not occupy the same time step as the US. If it does, because its timing is somehow delayed or because its momentum extends beyond US onset, CF inputs to the cerebellar cortex would be inhibited by the projection from IP to IO. Trials on which this occurs would be tantamount to extinction trials, thereby countering the down-regulated state of PF/ PC synapses that mediate CRs. PF/PC synapses expressing LTD would gradually lose this capacity until US-triggered CF inputs are no longer blocked by overly robust CRs. This feedback mechanism could be a vital aspect of CR timing, in that it would ensure that CRs do not become so robust that they lose precision in predicting US onsets.

The TD learning rule is not a simple competitive rule because of the $\gamma Y(t)$ term in equation 8 . As noted previously, the TD learning rule is implemented by a combination of two reinforcement components. The first is donated by the US and represented by $\lambda$ in equation 8 . The implementation assumes that $\lambda$ can be aligned with CF activation of PCs, which functions to produce LTD among coactive PF synapses, as depicted in Figure 1. The second reinforcement component is donated by the $\dot{Y}(t)$ terms in the learning rule, $\gamma Y(t)-Y(t-1)$. This information is conveyed to $\mathrm{HVI}$ by the projection from RN and SpO shown in Figure 1.

Figure 6 shows circuit elements, not shown in Figure 1 , for implementing the $\dot{Y}(t)$ component of the learning rule. These components include the projections to the cerebellar cortex from the $\mathrm{RN}$ and SpO indicated in Figure 1. We hypothesize that the $\mathrm{RN}$ projection carries information (feedback) about $Y(t)$ to the cerebellar cortex as efference copy. PFs project this information to PCs that have collaterals to a set of Golgi cells. Because these projections are inhibitory (Ito 1984), these PCs invert the efference signal from the RN. In addition, the interpositioning of the PCs between the RN and Golgi cells attenuates the signal and implements the TD model's discount factor $\gamma$.

Because Golgi cells are inhibitory on granule cells, the effect of their inhibition by PCs receiving efference from the $\mathrm{RN}$ would be to disinhibit ac-

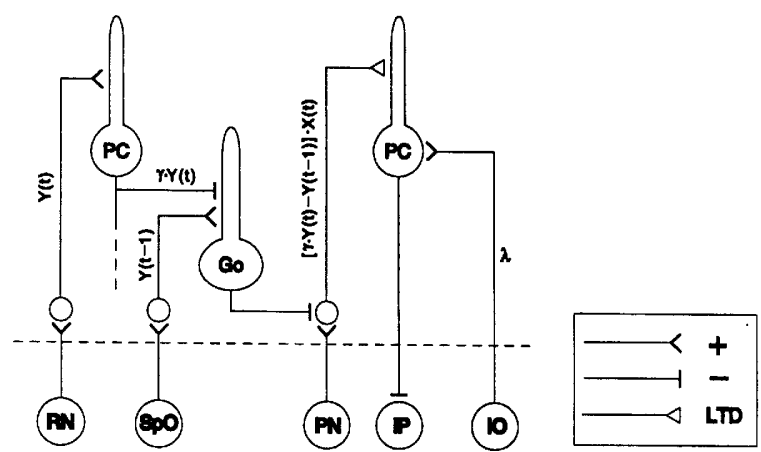

Figure 6: Neural circuits of the cerebellum implementing $\gamma Y(t)$ and other variables of the TD learning rule. (Go) Golgi cells.

tivity of granule cells. Because granule cells relay CS information from the PN to PCs involved in LTD and CR generation, disinhibition of granule cells by Golgi cells enhances the information flow from active CS components. Mathematically, the implementation assumes that the variables $X_{j}$ in equation 9 engage granule cells. PFs arising from these granule cells trigger output, and they affect connection weights residing at PF/PC synapses in proportion to $\dot{Y}(t) \times X_{j}$.

PCs driven by projections from the RN would increase their firing rate so as to mimic the representation of the CR as it passes through the RN enroute to MNs and SpO. Berthier and Moore (1986) recorded from several HVI PCs with CRrelated increases in firing. Because increases in firing during a CS is inconsistent with the LTD hypothesis of CR generation, these PCs serve some other function. One possibility is that they inhibit motor programs incompatible with CR generation. Here, we are suggesting an additional function of these PCs, that of projecting inverted and discounted CR efference from the RN to Golgi cells.

The implementation assumes that the Golgi cells that receive the inverted efference from the RN also receive a direct, noninverted, excitatory projection from $\mathrm{SpO}$. This projection carries information about the CR at time $t-\Delta t$. Therefore, the Golgi cell in Figure 6 fires at a rate determined by the differential between two inputs: $\gamma Y(t)$ donated by the RN and $Y(t-\Delta t)$ donated by SpO. ${ }^{6}$ Hence,

\footnotetext{
${ }^{6}$ The PC that donates $\gamma$ does not impose a significant delay in transmitting feedback from $\mathrm{RN}$ to Golgi cells. Conduction distances within the cerebellar cortex are on the order of tens of microns. Projections to the cerebellum from the brain stem, and from the $\mathrm{RN}$ to the SpO, are on the order of tens of millimeters.
}

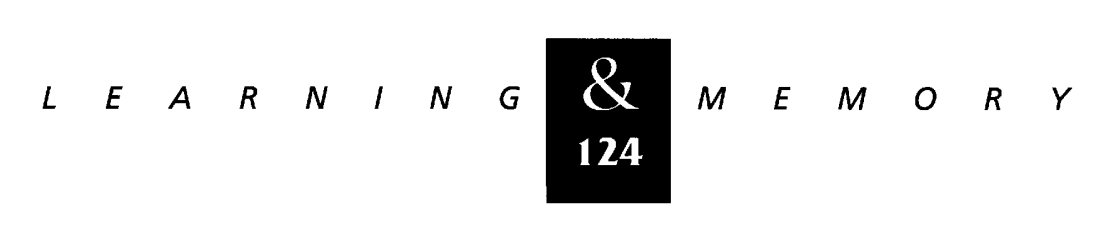


Golgi cells act as $\dot{Y}(t)$ detectors. In terms of equation $8, Y(t)$ is transmitted to cerebellar granule cells by the $\mathrm{RN}$, and $Y(t-1)$ is transmitted to granule cells from SpO. The RN input engages PCs that inhibit Golgi cells responsible for gating inputs from CSs to PCs. Efference from SpO engages the same Golgi cells directly. Because Golgi cells are inhibitory on granule cells, the bigger the $\mathrm{RN}$ input relative to SpO input is, the bigger the signal from serial component CSs active at that time, be they from onset or offset cascades.

In this way, the Golgi cells that implement $\dot{Y}(t)$ reinforce and maintain the down-regulated state of active $\mathrm{PF} / \mathrm{PC}$ synapses. $\mathrm{PF} / \mathrm{PC}$ synapses that are activated by a CS element are down-regulated by the contiguous US-triggered activation of CF input from the IO. As CS elements earlier in the timing sequence become capable of evoking an output that anticipates the US, inhibition is relayed to the olive and the US loses its capacity to trigger a CF volley (see Fig. 1). However, the down-regulation of these synapses is maintained, and still earlier CS elements are recruited, by PFs carrying $\dot{Y} \times X_{j}$ to LTD-PCs, as indicated in Figure $6 .^{7}$

In their recording study, Desmond and Moore (1991a) observed an average lead time of $36 \mathrm{msec}$ between the initiation of CR-related firing in RN neurons and the peripherally observed CR. Richards et al. (1991) observed an average lead time of $20 \mathrm{msec}$ in SpO neurons. Therefore, the time difference in CR-related efference arising from the two structures is on the order of $15-20 \mathrm{msec}$. This difference spans one 10-msec time step used in our simulations with the TD model. This temporal difference is consistent with a conduction velocity of $2 \mathrm{~m} / \mathrm{sec}$ for the 10-mm trajectory of unmyelinated axons from the RN to rostral portions of the SpO. The 10-msec grain also ensures good resolution of fast transients. The fastest transients in eye-blink conditioning occur during unconditioned responses. At its fastest, the eyelids require $80 \mathrm{msec}$ to close completely, with a peak velocity of $4-5$ $\mathrm{mm} / 20 \mathrm{msec}$.

${ }^{7}$ Hartell (1996) showed that strong PF activity can induce LTD at PF/PC synapses and that this depression extends to other, spatially separated synapses. Hartell (1996) also reported that LTD induced by strong PF stimulation occludes the form of LTD mediated by CFs. Schreurs et al. (1997) report a similar occlusion of CF-mediated LTD among PCS that express LTP. Hartell (1996) did not address the question of whether strong PF stimulation maintains and reinforces previous/y established CFinduced LTD, which is a core assumption of the implementation.

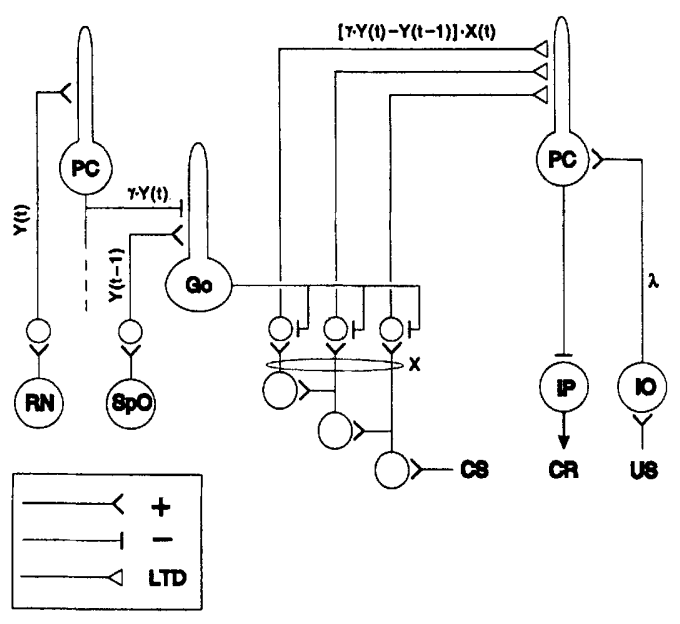

Figure 7: The complete TD implementation scheme showing three sequentially activated CS components, representing onset and offset cascades in the manner of the VET model of Desmond and Moore (1988). (Go) Golgi cells.

Efference from SpO neurons recorded among HVI PCs would tend to lag behind the peripherally observed CR, especially if it arises from more caudal portions of the structure. Berthier and Moore (1986) observed a continuum of lead and lag times among PCs that increased their firing to the CS. PCs that receive projections from the SpO (not shown in Fig. 6) would be expected to increase their firing but with a lag relative to those receiving projections from the RN. The proportion of CRleading PCs observed by Berthier and Moore (1986) matched the number of CR-lagging PCs, which makes sense if the two populations reflect CR efference from two spatially separated sources, $\mathrm{RN}$ and SpO.

Figure 7 is an expanded version of Figure 6 showing three sets of granule cells associated with three serial component CSs. These components might arise from CS onset or offset. The degree to which information from any of these serial CS components reaches the PCs to which they project is determined by Golgi cells firing in proportion to $\dot{Y}(t)$, as just described. Figure 2 shows that TDsimulated CRs tend to be positively accelerating (contingent upon $\gamma$ ) up to the occurrence of the US, so $\dot{Y}(t)$ increases progressively over the CS-US interval. Therefore, those PF/PC synapses activated nearest the time of the $\mathrm{CF}$ signal from the US have the greatest impact in establishing and maintaining LTD. This mechanism ensures the appropriate form and timing of CRs. 


\section{Interpretations of $\dot{Y}:$ Efference or Afference}

Equation 8 emphasizes interpretations of $\dot{Y}$ as efference, but it is equally correct to interpret changes in associative values in terms of afference, substituting equation 9 into equation 8 . Recent studies by Ramnani and Yeo (1996) and Hardiman et al. (1996) suggest that the efference interpretation of $\dot{Y}$ is correct. These studies show that temporary inactivation of IP by muscimol prevents extinction of the CR. That is, CS-alone trials that would normally lead to a gradual elimination of the CR instead had no effect whatsoever. When tested after the muscimol blockade had been removed, the previously established CR was at full strength but extinguished normally with continuing presentation of CS-alone trials. This finding is consistent with the efference interpretation of the TD implementation because inactivation of IP eliminates the $\mathrm{CR}$ and therefore prevents efference from the RN and $\mathrm{SpO}$ from reaching the putative site of learning in HVI. In terms of equation 8 , connection weights cannot decrease if $Y(t)$ and $Y(t-1)$ are both equal to 0 . If the afference interpretation were correct and efference plays no role in extinction, then inactivation of IP would not prevent extinction because afference arises from PN and bypasses IP enroute to HVI.

\section{Implications of the Implementation}

The implementation has several testable implications.

1. One implication is that some PCs decrease their firing rate in anticipation of $\mathrm{CR}$ peaks. These PCs express LTD. However, there are other PCs, perhaps the majority, that increase their rate of firing in relation to $\mathrm{CR}$ peaks, as reported by Berthier and Moore (1986). Some of these PCs express efference from the RN and SpO. Their function is to activate Golgi cells that modulate information flow through the granule cells. Another function would be to inhibit motor programs such as eye opening and saccadic movements that could interfere with eyelid closure.

2. The implementation specifies that Golgi cells that modulate the flow of CS information in granule cells fire in relation to changes in eyelid position, that is, they fire in relation to $\dot{Y}$. This property of Golgi cell-firing patterns has been reported by van Kan et al. (1993), in a study of monkey limb movements, and Edgley and Lidierth (1987), in a study of cat locomotion.

3 . The implementation implies that reversible inactivation of the $\mathrm{RN}$ would prevent second-order conditioning. However, although inactivation of the $\mathrm{RN}$ would cause a temporary interruption of information flow that results in a $C R$, it would not prevent learning of the primary association between components of the CS and the US. This association proceeds with little disruption because the PN and the IO can still convey CS and US information to the cerebellar cortex. Evidence for this proposition comes from a study of rabbit eye-blink conditioning by Clark and Lavond (1993). They demonstrated that inactivation of the RN by cooling did not prevent learning, as CR magnitude recovered upon reactivation of the RN. However, inactivation of the $\mathrm{RN}$ would interrupt efference about the position of the eyelid at times $t$ and $t-\Delta t$ from the $\mathrm{RN}$ and SpO. Thus, $Y$ would not be available to the cerebellar cortex. According to the TD model, $\dot{Y}$ allows for increments of predictive associations in the absence of the US, as would occur in second-order conditioning. This being the case, inactivation of the $\mathrm{RN}$ would interfere with second-order conditioning. With the RN inactivated, animals trained with a mixture of first- and second-order (Kehoe et al. 1981) would be expected to show first-order learning, as in the Clark and Lavond (1993) study, but little or no second-order learning. Figure $\mathbf{8}$ shows a simulation of the failure of second-order conditioning by blocking the $Y(t)$ efference projection to HVI from the RN. ${ }^{8}$

\section{Summary}

We have described how the TD model of classical conditioning can generate appropriate CR waveforms in simple protocols and complex paradigms involving temporal uncertainty. We also suggest an implementation scheme for TD learning within the cerebellum. The implementation draws on neurobiological evidence regarding how LTD is

\footnotetext{
${ }^{8}$ Mechanisms for establishing second-order conditioning within the cerebellum are unknown. A TD implementation based on LTD assumes that PF inputs arising from second-order serial components express LTD even though the US is withheld. Hartell (1996) provides evidence favorable for such a mechanism.
}

\begin{tabular}{lllllllllllllll}
\hline & $A$ & $R$ & $N$ & $I$ & $N$ & $G$ & $\mathbf{Q}_{126}$ & $M$ & $E$ & $M$ & $O$ & $R$ & $Y$
\end{tabular}


A
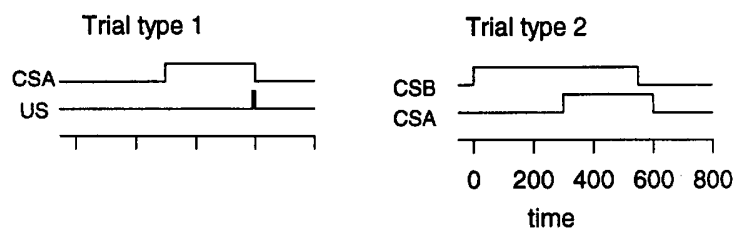

B

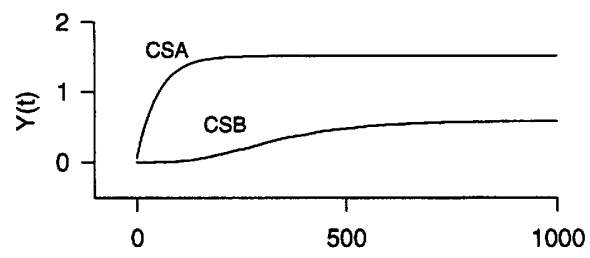

C

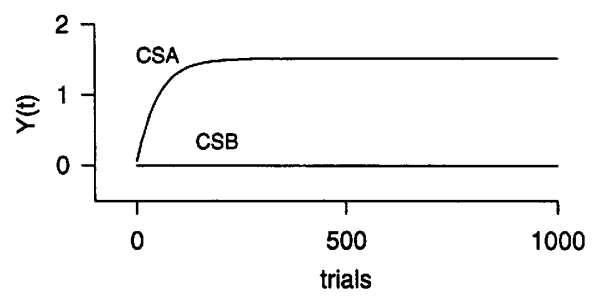

Figure 8: Simulated first- and second-order conditioning after Kehoe et al. (1981). (A) The training protocol. Trial-type 1: CSA is $300 \mathrm{msec}$ in duration, and the CSUS interval is $290 \mathrm{msec}$. Trial type 2: CSB is $550 \mathrm{msec}$ in duration, and the CSB-CSA interval is $300 \mathrm{msec}$. The two trial types are randomly mixed, and each occurs 500 times. $(B)$ Simulated peak CR amplitude $[Y(t)]$ to CSA and CSB as a function of trials. Note that $Y(t)$ to CSB increases with training, reflecting second-order conditioning. (C) With $\gamma=0$ (equivalent to inactivation of the $\mathrm{RN}$ ), second-order conditioning does not occur.

established, reinforced, and maintained among PCs that determine the timing and topography of CRs. The implementation incorporates recent anatomical findings, reviewed by Rosenfield and Moore (1995), that allow these PCs to receive the two components of the TD model's reinforcement operator, one component donated by the US and another component donated by $\dot{Y}(t)=Y(t)-$ $Y(t-\Delta t)$ as feedback efference. The implementation lays the foundation for network simulations at the cellular level.

\section{Acknowledgments}

We thank the following individuals for helpful comments on various aspects of this article: Eli Brandt, Peter Dayan, Bernard Schreurs, and an anonymous reviewer.

\section{References}

Berthier, N.E. and J.W. Moore. 1986. Cerebellar Purkinje cell activity related to the classically conditioned nictitating membrane response. Exp. Brain Res. 63: 341-350.

1990. Activity of deep cerebellar nuclear cells during classical conditioning of nictitating membrane extension in rabbits. Exp. Brain Res. 83: 44-54.

Berthier, N.E., A.G. Barto, and J.W. Moore. 1991. Linear systems analysis of the relationship between firing of deep cerebellar neurons and the classically conditioned nictitating membrane response in rabbits. Biol. Cybern. 65: 99-105.

Blazis, D.E.J. and J.W. Moore. 1991. Conditioned inhibition of the nictitating membrane response in rabbits following hypothalamic and mesencephalic lesions. Behav. Brain Res. 46: $71-81$.

Bullock, D, J.C. Fiala, and S. Grossberg. 1994. A neural model of timed response learning in the cerebellum. Neural Networks 7: 1101-1114.

Chen, C. and R.F. Thompson. 1995. Temporal specificity of long-term depression in parallel fiber-Purkinje synapses in rat cerebellar slice. Learn. \& Mem. 2: 185-198.

Clark, R.E. and D.G. Lavond. 1993. Reversible lesions of the red nucleus during acquisition and retention of a classically conditioned behavior in rabbits. Behav. Neurosci.

107: 264-270.

Desmond, J.E. 1990. Temporally adaptive responses in neural models: The stimulus trace. In Learning and computational neuroscience: Foundations of adaptive networks (ed. M. Gabriel and J. Moore), pp. 421-461. MIT Press, Cambridge, MA.

Desmond, J.E. and J.W. Moore. 1988. Adaptive timing in neural networks: The conditioned response. Biol. Cybern. 58: $405-415$.

1991a. Activity of red nucleus neurons during the classically conditioned rabbit nictitating membrane response. Neurosci. Res. 10: 260-279.

1991b. Altering the synchrony of stimulus trace processes: Tests of a neural-network model. Biol. Cybern. 65: $161-169$.

Edgley, S.A. and M. Lidierth. 1987. Discharges of cerebellar Golgi cells during locomotion in cats. J. Physiol. (Lond.) 392: $315-332$.

Eilers, J., G.J. Augustine, and A. Konnerth. 1995. Subthreshold synaptic $\mathrm{Ca}^{2+}$ signaling in fine dendrites and spines of cerebellar Purkinje neurons. Nature 373: 155-158.

Fiala, J.C., S. Grossberg, and D. Bullock. 1996. Metabotropic

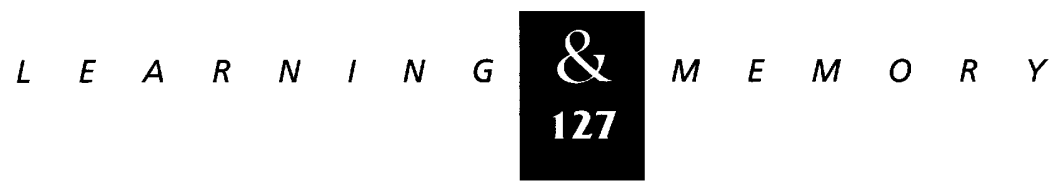


glutamate receptor activation in cerebellar Purkinje cells as substrate for adaptive timing of the classically conditioned eye-blink response. J. Neurosci. 16: 3760-3774.

Ghosh, A. and M.E. Greenberg. 1995. Calcium signaling in neurons: Molecular mechanisms and cellular consequences. Science 268: 239-247.

Grossberg, S. and J.W.L. Merrill. 1996. The hippocampus and cerebellum in adaptively timed learning, recognition, and movement. J. Cognit. Neurosci. 8: 257-277.

Gruart, A. and C.H. Yeo. 1995. Cerebellar cortex and eyeblink conditioning: Bilateral regulation of conditioned responses. Exp. Brain Res. 104: 431-448.

Hardiman, M.J., N. Ramnani, and C.H. Yeo. 1996. Reversible inactivations of the cerebellum with muscimol prevent the acquisition and extinction of conditioned nictitating membrane responses in the rabbit. Exp. Brain Res. 110: $235-247$.

Hartell, N.A. 1996. Strong activation of parallel fibers produces localized calcium transients and a form of LTD that spreads to distant synapses. Neuron 16: 601-610.

Hesslow, G. 1994. Inhibition of classically conditioned eyeblink responses by stimulation of the cerebellar cortex in the cat. J. Physiol. 476: 245-256.

Ito, M. 1984. The cerebellum and neural control. Raven Press, New York, NY.

Kano, M., U. Rexhausen, J. Dreessen, and A. Konnerth. 1992. Synaptic excitation produces a long-lasting rebound potentiation of inhibitory synaptic signals in cerebellar Purkinje cells. Nature 356: 601-604.

Kehoe, E.J., A.M. Feyer, and J.L. Moses. 1981. Second-order conditioning of the rabbit's nictitating membrane response as a function of the CS2-CS1 and CS1-US intervals. Anim. Learn. \& Behav. 9: 304-315.

Kim, J.J. and R.F. Thompson. 1997. Cerebellar circuits and synaptic mechanisms involved in classical eyeblink conditioning. Trends Neurosci. 20: 177-181.

Klopf, A.H. 1988. A neuronal model of classical conditioning. Psychobiology 16: 85-125.

Konnerth, A., J. Dreessen, and G.T. Augustine. 1992. Brief dendritic signals initiate long-lasting synaptic depression in cerebellar Purkinje ceils. Proc. Natl. Acad. Sci.

89: 7051-7055.

Millenson, J.R., E.J. Kehoe, and I. Gormezano. 1977. Classical conditioning of the rabbit's nictitating membrane response under fixed and mixed CS-US intervals. Learn. \& Motiv. 8: 351-366.

Moore, J.W. 1992. A mechanism for timing conditioned responses. In Time, action, and cognition (ed. F. Macar, V.
Pouthas, and W.J. Friedman), pp. 229-238. Kluywer Academic Publishers, Dordrecht, The Netherlands.

Moore, J.W. and J.E. Desmond. 1992. A cerebellar neural network implementation of a temporally adaptive conditioned response. In Learning and memory: The behavioral and biological substrates (ed. 1. Gormezano and E.A. Wasserman) pp. 347-368. Lawrence Erlbaum Associates, Hillsdale, NJ.

Moore, J.W., J.E. Desmond, N.E. Berthier, D.E.J. Blazis, R.S. Sutton, and A.G. Barto. 1986. Simulation of the classically conditioned nictitating membrane response by a neuron-like adaptive element: Response topography, neuronal firing, and interstimulus intervals. Behav. Brain Res. 21: 143-154.

Moore, J.W., J.E. Desmond, and N.E. Berthier. 1989. Adaptively timed conditioned responses and the cerebellum: A neural network approach. Biol. Cybern. 62: 17-28.

Perrett, S.P. and M.D. Mauk. 1995. Extinction of conditioned eyelid responses requires the anterior lobe of cerebellar cortex. J. Neurosci. 15: 2074-2080.

Perrett, S.P., B.P. Ruiz, and M.D. Mauk. 1993. Cerebellar cortex lesions disrupt learning-dependent timing of conditioned eyelid responses. J. Neurosci. 13: 1708-1718.

Ramnani, N. and C.H. Yeo. 1996. Reversible inactivations of the cerebellum prevent the extinction of conditioned nictitating membrane responses in rabbits. J. Physiol. 495: $159-168$.

Raymond, J.L., S.G. Lisberger, and M.D. Mauk. 1996. The cerebellum: A neuronal learning machine? Science 272: 1126-1131.

Rescorla, R.A. and A.R. Wagner. 1972. A theory of Pavlovian conditioning: Variations in the effectiveness of reinforcement and nonreinforcement. In Classical conditioning II: Current theory and research (ed. A.H. Black and W.F. Prokasy), pp. 64-99. Appleton-Century-Crofts, New York, NY.

Richards, W.G., T.N. Ricciardi, and J.W. Moore. 1991. Activity of spinal trigeminal pars oralis and adjacent reticular formation units during differential conditioning of the rabbit nictitating membrane response. Behav. Brain Res. 44: 195-204.

Rosenfield, M.E. and J.W. Moore. 1995. Connections to cerebellar cortex (Larsell's HVI) in the rabbit: A WGA-HRP study with implications for classical eyeblink conditioning. Behav. Neurosci. 109: 1106-1118.

Schreurs, B.G. and D.L. Alkon. 1993. Rabbit cerebellar slice analysis of long-term depression and its role in classical conditioning. Brain Res. 631: 235-240.

Schreurs, B.G., M.M. Oh, and D.L. Alkon. 1996. Pairing-specific long-term depression of Purkinje cell excitatory postsynaptic potentials results from a classical conditioning procedure in the rabbit cerebellar slice. $J$. Neurophysiol. 75: 1051-1060.

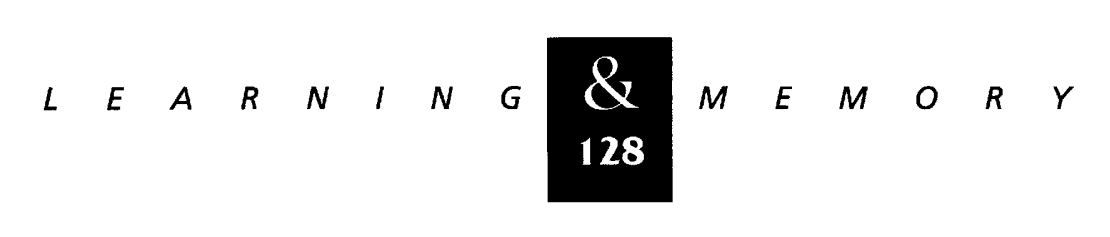


Schreurs, B.G., D. Tomsic, P.A. Gusev, and D.L. Alkon. 1997. Dendritic excitability microzones and occluded long-term depression after classical conditioning of the rabbit's nictitating membrane response. J. Neurophysiol. 77: 86-92.

Schultz, W., P. Dayan, and P.R. Montague. 1997. A neural substrate of prediction and reward. Science 275: 1593-1599.

Sears, L.L. and J.E. Steinmetz. 1991. Dorsal accessory inferior olive activity diminishes during acquisition of the rabbit classically conditioned eyelid response. Brain Res.

545: 114-122.

Sutton, R.S. and A.G. Barto. 1981. Toward a modern theory of adaptive networks: Expectation and prediction. Psychol. Rev. 88: 135-171.

1990. Time-derivative models of Pavlovian reinforcement. In Learning and computational neuroscience: Foundations of adaptive networks (ed. M. Gabriel and J. Moore), pp. 497-537. MIT Press, Cambridge, MA.

Thompson, R.F. 1986. The neurobiology of learning and memory. Science 233: 941-947.

Thompson, R.F. and D.J. Krupa. 1994. Organization of memory traces in the mammalian brain. Annu. Rev. Neurosci. 17: 519-549.

van Kan, P.L.E., A.R. Gibson, and J.C. Houk. 1993. Movement-related inputs to intermediate cerebellum of the monkey. J. Neurophysiol. 69: 74-94.

Wells, G.R., M.J. Hardiman, and C.H. Yeo. 1989. Visual projections to the pontine nuclei of the rabbit: Orthograde and retrograde tracing studies with WGA-HRP. J. Comp. Neurol. 279: 629-652.

Received February 18, 1997; revised version accepted April 30, 1997. 


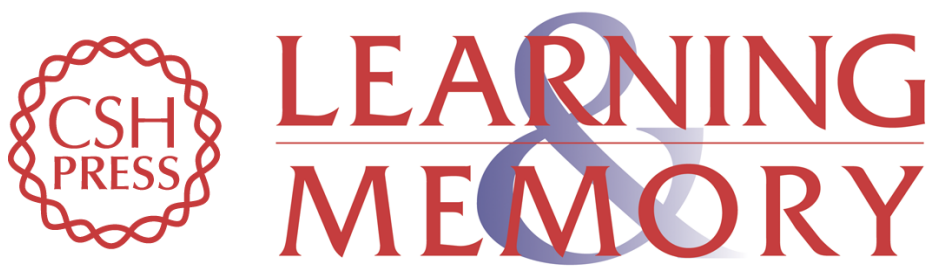

\section{Conditioned response timing and integration in the cerebellum.}

J W Moore and J S Choi

Learn. Mem. 1997, 4:

Access the most recent version at doi:10.1101//m.4.1.116

References This article cites 43 articles, 9 of which can be accessed free at: http://learnmem.cshlp.org/content/4/1/116.full.html\#ref-list-1

License

Email Alerting Receive free email alerts when new articles cite this article - sign up in the box at the Service top right corner of the article or click here. 